INFNCA-TH9514

LPC 9524

hep-ph/9511432

\title{
Glueball plus pion production in hadron collisions
}

\author{
Francesco Murgia \\ Istituto Nazionale di Fisica Nucleare, Sezione di Cagliari \\ via Ada Negri 18, I-09127 Cagliari, Italy \\ Paul Kessler and Joseph Parisi \\ Laboratoire de Physique Corpusculaire, Collège de France \\ 11, Place Marcelin Berthelot F-75231 Paris Cedex 05, France
}

(November 1995)

\begin{abstract}
Using a non-relativistic gluon bound-state model for glueballs $(\mathrm{G})$, we compute the subprocess $q \bar{q} \rightarrow G \pi$, and we therefrom derive the yield of the overall reaction $p \bar{p} \rightarrow G \pi X$, assuming the glueball and the pion to be emitted with their transverse momenta large, opposite and approximately equal. Numerical results are presented in the form of $p_{T}$ spectra for various glueball candidates and their possible quantum states, assuming those particles to be produced, in the type of reactions here considered, at high-energy $p \bar{p}$ colliders such as the CERN Sp̄pS.
\end{abstract}




\section{INTRODUCTION}

A non-relativistic gluon bound-state model for computing the production and decay of glueballs (G) made of two gluons was proposed a few years ago by Kada et al. [1], who used it in order to calculate the processes $J / \psi \rightarrow G \gamma$ and $G \rightarrow \gamma \gamma$. That model was later generalized for more complex reactions by Houra-Yaou et al. [2], and applied by them to the production of a glueball plus a quark or gluon jet at high transverse momentum in hadron collisions. Another application, recently computed by Ichola and Parisi [3], concerned glueball plus pion production in two-photon processes. In this paper we consider the production of the same final state as in Ref. [3], but this time in hadron collisions.

Indeed, while the existence of glueballs is considered a crucial test of quantum chromodynamics [4], and after a few glueball candidates have emerged in the early eighties from various experiments [5], further experimental evidence appears still necessary in order to firmly establish their nature and properties. Besides other reactions that should involve a "gluon-rich environment" (such as radiative $J / \psi$ decays, as well as diffractive hadronhadron scattering assumed to involve double Pomeron exchange), hard collisions occurring in high-energy reactions may provide another means of creating that kind of environment and thus producing glueballs.

We are aware that the status of the three particles that were considered as glueball candidates in Refs. [1-3], namely the $\eta(1440)$, the $f_{2}(1720)$, and the $X(2220)$, has become more uncertain in the last few years [6 8]. However, as has been discussed at large in Ref. [3], recent experimental data regarding those particles are rather contradictory; actually there has also been recently some positive evidence regarding the $f_{2}(1720)$ [9] and the $X(2220)$ [10]. Anyway, for none of the three candidates it has been decisively proved that it should not be a glueball. Therefore, in this paper, we still stick to the assumptions of Refs. [1] 3 .

Hereafter, in section II, we recall the formalism used and present the details of our calculation. Section III contains a discussion of the numerical results obtained and a brief conclusion. Two appendices provide respectively the expressions of all quantities (fourmomenta, polarization four-vectors, projectors of spinor pairs) needed for our calculation and those of all independent helicity amplitudes obtained for the subprocess $q \bar{q} \rightarrow G\left(q^{\prime} \bar{q}^{\prime}\right)_{P S}$ resp. $q \bar{q} \rightarrow G \pi$.

\section{DESCRIPTION OF THE FORMALISM AND DETAILS OF CALCULATION}

Let us first remark that, for $G \pi$ production in hadronic reactions, the hard process is necessarily induced by quark-antiquark collisions. Indeed, the subprocess $g g \rightarrow G \pi^{0}$ is excluded since, due to isospin conservation, the pion cannot be coupled to any parton system composed exclusively of gluons. For the same reason, the subprocess $q \bar{q} \rightarrow G \pi^{0}$ cannot involve any Feynman diagram where the quark and antiquark annihilate into a gluon. Therefore the calculation of the hard subprocess is the same for $G \pi^{0}$ and $G \pi^{ \pm}$production; at lowest order in perturbative QCD it involves the diagrams shown in Fig. 1, where the gluons $\left(g_{1}, g_{2}\right)$ and the final quarks $\left(q^{\prime}, \bar{q}^{\prime}\right)$ are, respectively, the components of the glueball and the pion to be produced. 
Applying the non-relativistic gluon bound-state model [1] for glueballs, together with the well known Brodsky-Lepage model [11] for pions, we write, in analogy with Eq. (1) of Ref. [3]:

$$
\begin{aligned}
\mathcal{M}_{q \bar{q} \rightarrow G\left(q^{\prime} \bar{q}^{\prime}\right)_{P S}}^{\lambda \bar{\lambda}, \Lambda}(E, \Theta, z) & =f_{L} \lim _{\beta \rightarrow 0} \frac{1}{\beta^{L}} \int \frac{d(\cos \theta) d \varphi}{4 \pi} \\
& \times \sum_{\lambda_{1}, \lambda_{2}} \zeta_{\lambda_{1} \lambda_{2}}^{L S \Lambda}(\theta, \varphi) \mathcal{M}_{q \bar{q} \rightarrow g_{1} g_{2}\left(q^{\prime} \bar{q}^{\prime}\right)_{P S}}^{\lambda \bar{\lambda}, \lambda_{1} \lambda_{2}}(E, \Theta, \theta, \varphi, z)
\end{aligned}
$$

where we have used the following definitions: $E$ and $\Theta$ are, respectively, the total energy and the pion emission angle in the $q \bar{q}$ center-of-mass frame, while $\theta(\varphi)$ is the polar (azimuthal) emission angle of either gluon in the $g_{1} g_{2}$ c.m. frame, i.e. the glueball rest frame (see Fig. 2). $z$ is the Brodsky-Lepage variable defining the fractional momentum of the quark $q^{\prime}$ within the pion. We call $J, L, S, \Lambda$ respectively the total spin of the glueball, its orbital angular momentum, its intrinsic spin, and the component of its total spin along the $z$-axis of Fig. 2. In addition, we call $\lambda(\bar{\lambda})$ the helicities of $q(\bar{q})$, while $\lambda_{1}\left(\lambda_{2}\right)$ are the helicities of $g_{1}\left(g_{2}\right)$ in the glueball rest frame. The angular projection function $\zeta_{\lambda_{1} \lambda_{2}}^{L S J \Lambda}(\theta, \varphi)$ is defined as

$$
\zeta_{\lambda_{1} \lambda_{2}}^{L S J \Lambda}(\theta, \varphi)=d_{\Lambda \bar{\Lambda}}^{J}(\theta) e^{-i \Lambda \varphi}\langle L S 0 \bar{\Lambda} \mid L S J \bar{\Lambda}\rangle\left\langle 11 \lambda_{1},-\lambda_{2}\right| \begin{array}{llll}
1 & 1 & S & \Lambda
\end{array}
$$

where $\bar{\Lambda}=\lambda_{1}-\lambda_{2}$. $\beta$ is the velocity of either gluon in the glueball rest frame, while $f_{L}$ is given by

$$
f_{L}=\sqrt{\frac{2 L+1}{2 \pi M}}\left(-\frac{2 i}{M}\right)^{L} \frac{(2 L+1) ! !}{L !}\left[\left(\frac{d}{d r}\right)^{L} R_{L}(r)\right]_{r=0}
$$

where $M$ is the glueball mass and $R_{L}(r)$ its radial wave function in configuration space. Finally we notice that the system $q^{\prime} \bar{q}^{\prime}$ is here assumed to be in a pseudoscalar (PS) state.

In the following stage we apply the Brodsky-Lepage convolution formula [11:

$$
\mathcal{M}_{q \bar{q} \rightarrow G \pi}^{\lambda \bar{\lambda}, \Lambda}(E, \Theta)=\int d z \Phi_{\pi}(z) \mathcal{M}_{q \bar{q} \rightarrow G\left(q^{\prime} \bar{q}^{\prime}\right)_{P S}}^{\lambda \bar{\lambda}, \Lambda}(E, \Theta, z)
$$

where $\Phi_{\pi}(z)$ is the pion distribution amplitude.

As in Refs. [2, 3] we assume the glueball to be extreme-relativistic in the $q \bar{q}$ c.m. frame, i.e. $M / E \rightarrow 0$. In that approximation the gluons are also treated as massless in the hard subprocess. A fortiori the mass of the pion, as well as of its constituent quarks, is also neglected. In other words: both outgoing particles, and all partons involved, are on the light cone.

It is to be noticed that, with massless quarks, helicity conservation 11 imposes: $\bar{\lambda}=-\lambda$. On the other hand, due to parity and angular-momentum conservation, one has the relation $\mathcal{M}_{q \bar{q} \rightarrow G \pi}^{-\lambda,-\bar{\lambda},-\Lambda}=(-1)^{J+L+\Lambda+1} \mathcal{M}_{q \bar{q} \rightarrow G \pi}^{\lambda \bar{\lambda}, \Lambda}$, which reduces the number of independent amplitudes by an additional factor of two. It thus becomes sufficient to limit oneself to computing those amplitudes where $\lambda=1 / 2, \bar{\lambda}=-1 / 2$.

In Appendix A we show the expressions of four-momenta, polarization four-vectors and spinors needed for our calculation. Appendix B contains the expressions of the independent 
helicity amplitudes obtained, corresponding to the various glueball quantum states considered, both after applying Eq. (1) and after we use Eq. (画) involving a convolution with the pion distribution amplitude $\Phi_{\pi}(z)$. For the latter we choose two different expressions, namely that proposed by Chernyak and Zhitnitsky [12]:

$$
\Phi_{\pi}^{C Z}(z)=5 \sqrt{3} f_{\pi} z(1-z)(2 z-1)^{2}
$$

and the so-called asymptotic one [11]

$$
\Phi_{\pi}^{a s}(z)=\sqrt{3} f_{\pi} z(1-z)
$$

where $f_{\pi}$ is the pion decay constant $\left(f_{\pi} \cong 93 \mathrm{MeV}\right)$.

From the amplitudes thus obtained one derives the transverse-momentum spectrum for the subprocess considered, taking account of kinematic factors (where again one makes $M / E \rightarrow 0):$

$$
\begin{aligned}
\frac{d \sigma^{q \bar{q} \rightarrow G \pi}}{d p_{T}}\left(E, p_{T}\right) & =\frac{p_{T}}{288 \pi E^{3} \sqrt{E^{2}-4 p_{T}^{2}}} \\
& \times \sum_{i, j} \sum_{\lambda, \bar{\lambda}, \Lambda}\left[\left|\mathcal{M}_{q \bar{q} \rightarrow G \pi}^{\lambda \bar{\lambda}, \Lambda}(E, \Theta)\right|^{2}+\left|\mathcal{M}_{q \bar{q} \rightarrow G \pi}^{\lambda \bar{\lambda}, \Lambda}(E, \pi-\Theta)\right|^{2}\right]
\end{aligned}
$$

where, in the expressions of the amplitudes, $\cos \Theta$ is to be replaced by $\left(1-4 p_{T}^{2} / E^{2}\right)^{1 / 2}$ and $\sin \Theta$ by $2 p_{T} / E ; i, j$ are the color indices of $q, \bar{q}$ respectively.

The transverse-momentum spectrum for the overall reaction $p \bar{p} \rightarrow G \pi X$ is then given by convoluting the spectrum defined by Eq. (7) with the distribution functions of the quarks and the antiquarks inside the proton and the antiproton, as follows:

$$
\frac{d \sigma^{p \bar{p} \rightarrow G \pi X}}{d p_{T}}\left(s, p_{T}\right)=\int_{x_{\min }}^{1} d x \int_{x_{\min }^{\prime}}^{1} d x^{\prime} F\left(x, x^{\prime}, " Q^{2} "\right) \frac{d \sigma^{q \bar{q} \rightarrow G \pi}}{d p_{T}}\left(E, p_{T}\right)
$$

where $s$ is the overall c.m. energy squared; noticing that $E^{2}=x x^{\prime} s$, one gets: $x_{\min }^{\prime}=$ $E_{\text {min }}^{2} /(x s), x_{\text {min }}=E_{\text {min }}^{2} / s$, with $E_{\text {min }}=2 p_{T}$. As for the function $F\left(x, x^{\prime}\right.$, " $Q^{2}$ " $)$, it is defined in the following way:

(i) For $G \pi^{ \pm}$production

$$
F\left(x, x^{\prime}, " Q^{2 "}\right)=f_{u / p}\left(x, " Q^{2 "}\right) f_{d / p}\left(x^{\prime}, " Q^{2 "}\right)+f_{\bar{d} / p}\left(x, " Q^{2 "}\right) f_{\bar{u} / p}\left(x^{\prime}, " Q^{2 "}\right)
$$

(ii) For $G \pi^{0}$ production

$$
\begin{aligned}
F\left(x, x^{\prime}, " Q^{2 "}\right) & =\frac{1}{2}\left[f_{u / p}\left(x, " Q^{2 "}\right) f_{u / p}\left(x^{\prime}, " Q^{2 "}\right)+f_{d / p}\left(x, " Q^{2 "}\right) f_{d / p}\left(x^{\prime}, " Q^{2 "}\right)\right. \\
& \left.+f_{\bar{u} / p}\left(x, " Q^{2 "}\right) f_{\bar{u} / p}\left(x^{\prime}, " Q^{2} "\right)+f_{\bar{d} / p}\left(x, " Q^{2 "}\right) f_{\bar{d} / p}\left(x^{\prime}, " Q^{2} "\right)\right]
\end{aligned}
$$


Here we have made use of the fact that the quark (antiquark) content of the antiproton is equal to the antiquark (quark) content of the proton.

For the distribution functions $f_{q / p}, f_{\bar{q} / p}$ we use the parametrization CTEQ3 (leading order QCD) [13], while for the scale parameter we take " $Q 2$ " $=M^{2}$.

In order to eliminate the normalization constant $f_{L}^{2}$ (see Eq. (1)), we use the same procedure as in Refs. [1] 3], i.e. we write:

$$
\frac{d \sigma^{p \bar{p} \rightarrow G \pi X}}{d p_{T}} B(G \rightarrow x y \ldots)=\frac{d \sigma^{p \bar{p} \rightarrow G \pi X}}{d p_{T}} \frac{\Gamma(J / \psi \rightarrow G \gamma) B(G \rightarrow x y \ldots)}{\Gamma(J / \psi \rightarrow G \gamma)}
$$

where $B(G \rightarrow x y \ldots)$ is the branching ratio for glueball decay in a given channel (actually we shall consider only the main decay channel for each glueball candidate). Then the numerator in the second factor on the right-hand side of Eq. (11) is given by experimental measurements, while for the corresponding denominator we use the expressions computed before 11 | 1 .

Then, in principle, there is no free parameter left; yet there is a certain freedom of choice regarding the expressions of the $\alpha_{s}$ factors present in the calculation. Notice that on the r.h. side of Eq. (11) we get a factor $\alpha_{s}^{4}$ (coming from the helicity amplitudes, see Eqs. (7),(8) and appendix B), divided by a factor $\alpha_{s}^{2}$ contained in the $J / \psi$ partial decay width. Assuming that $\alpha_{s}$ takes approximately the same value in both processes considered, i.e. $p \bar{p} \rightarrow G \pi X$ and $J / \psi \rightarrow G \gamma$, we are left with a factor $\alpha_{s}^{2}$ in the final expression of the transverse-momentum spectra. We take: $\alpha_{s}\left(\right.$ " $Q^{2}$ ") $)=\alpha_{s}\left(M^{2}\right)=12 \pi /\left[25 \ln \left(M^{2} / \Lambda^{2}\right)\right]$, with $\Lambda=0.2 \mathrm{GeV}$.

The $p_{T}$ spectra thus obtained for the reaction $p \bar{p} \rightarrow G \pi^{0} X$ are shown, for the three glueball candidates and their respective quantum states here considered (the same as in Ref. [1 3], apart from a slight numerical modification ${ }^{* *}$, with either pion distribution amplitude assumed (Eqs. (5),(6)), at an $s$ value of $4 \cdot 10^{5} \mathrm{GeV}^{2}$ (chosen so as to be approximately

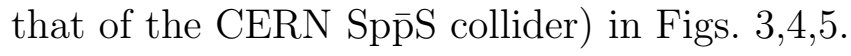

* The decay widths given in Ref. [1] have been systematically multiplied by a factor of 4 , since the helicity amplitudes had been underestimated there by a factor of 2 . In addition two misprints that appeared there, regarding the widths of $J / \psi$ radiative decay into glueballs with quantum states $J=0, L=S=1$ and $J=4, L=S=2$, have been corrected. Furthermore we have slightly modified the values of the numerator of the second factor on the r.h. side of Eq. (11), in accordance with the most recent experimental data (see Refs. [8,10]); in addition, for the $\eta(1440)$, we have here considered its decay in the $\rho^{0} \rho^{0}$ (instead of $K \bar{K} \pi$ ) channel, and for the $X(2220)$ its decay in the $\pi^{+} \pi^{-}$(instead of $\left.K \bar{K}\right)$ channel.

** The state called " $L=m$ " in Refs. [1] 3] is a mixture of states $L=0, S=2$ and $L=2, S=0$ with their respective weight coefficients connected by the relation $A_{20}=0.286 M^{2}\left[R_{0}(0) / R_{2}^{\prime \prime}(0)\right] A_{02}$, while the state called " $L=2$ " is a mixture of states $L=2, S=0$ and $L=2, S=2$ with their relative weight coefficients related by $A_{20}=-6.816 A_{22}$. Both mixtures have been adjusted in such a way that they fit the experimental ratios of helicity amplitudes measured in the process $J / \psi \rightarrow \gamma f_{2}(1720)$. 
As is shown by Fig. 6, taking the case of the $\eta(1440)$ as an example, there is very little difference between the respective $p_{T}$ spectra of neutral and charged pions produced together with glueballs; the latter are slightly lower.

\section{DISCUSSION AND CONCLUSION}

Our results call for the following remarks:

(i) The curves shown in Figs. 3, 4, 5 are approximately flat, i.e. the $p_{T}$ spectra obtained roughly scale like $p_{T}^{-7}$, as could be predicted from dimensional counting rules. Violations of that scaling rule are only logarithmic.

(ii) Correspondingly, it is only through logarithmic factors (of the type $\ln \left[s /\left(4 p_{T}^{2}\right)\right]$ or $\left.\ln ^{2}\left[s /\left(4 p_{T}^{2}\right)\right]\right)$ that those $p_{T}$ spectra depend on the machine energy. It results, as we have checked, that there is a slight increase, accompanied by a flattening of the curves, when one passes from SppsS to Tevatron energy, i.e. from $s=4 \cdot 10^{5} \mathrm{GeV}^{2}$ to $s=4 \cdot 10^{6}$ $\mathrm{GeV}^{2}$.

(iii) As usual, the yields predicted with the Chernyak-Zhitnitsky distribution amplitude are somewhat higher (by a factor of 3-4) than those computed with the asymptotic one.

(iv) If one integrates the spectra over $p_{T}$ from $p_{T_{m i n}}=5 \mathrm{GeV}$ on (assuming that there are no additional drastic acceptance cuts), the integrated cross sections obtained are of the order of $10^{-35}$ to $10^{-39} \mathrm{~cm}^{2}$, depending on the glueball candidate and quantum state considered, as well as on the pion distribution amplitude chosen. Some of those cross sections, i.e. those corresponding to the $\eta(1440)$, to the states " $L=2$ " and " $L=m$ " of the $f_{2}(1720)$ and to the state $J=4$ of the $X(2220)$, might be measurable under present experimental conditions. This conclusion calls however for some reservations, if the sources of uncertainty listed hereafter in (v)-(vii) are taken into account.

(v) In our calculation we have retained only lowest order terms in both the series expansion in powers of $M / E$ and that in powers of $\alpha_{s}$. Noticing that $M / E \lesssim 0.2$ (since $E_{\text {min }}=$ $\left.2 p_{T_{\min }}=10 \mathrm{GeV}\right)$ and $\alpha_{s}\left(M^{2}\right)=0.30 \div 0.35$, it still seems reasonable to expect that the inclusion of higher-order corrections would not modify the orders of magnitude obtained.

(vi) The parametrization of the quark distribution functions at very small values of $x, x^{\prime}$ (here $x_{\text {min }}=x_{\text {min }}^{\prime}=2.5 \cdot 10^{-4}$ ) has not yet been verified experimentally. As is shown in Fig. 0, a different parametrization such as MSRA [14] would lead to different shapes of the $p_{T}$ spectra and consequently to significantly lower values of the integrated cross sections.

(vii) One may also change the scale " $Q$ " " both in the expressions of the distribution functions and in that of the $\alpha_{s}$ factors involved in the calculation of $q \bar{q} \rightarrow G \pi$. For instance, setting " $Q$ " " $=p_{T}^{2}$ instead of $M^{2}$ modifies the $p_{T}$ spectra rather drastically and thus sharply affects, as well, the integrated cross sections (see Fig. 8). 
(viii) It is interesting to compare the yields here obtained with those of other exclusive channels in hadronic reactions, such as for instance pion pair production in $p \bar{p}$ collisions. That process has been computed, using the Brodsky-Lepage model [1], by Djagouri et al. [15] who have however restricted their calculation to a scattering angle of $90^{\circ}$ in the c.m. frame of the hard subprocess. Comparing $[d \sigma / d(\cos \Theta)]_{\Theta=90^{\circ}}$ for $p \bar{p} \rightarrow G \pi^{0} X$ and $p \bar{p} \rightarrow \pi^{0} \pi^{0} X$ under the same experimental conditions $\left(s=4 \cdot 10^{5}\right.$ $\mathrm{GeV}^{2}, p_{T}>5 \mathrm{GeV}$ ) and with the same theoretical ingredients (CTEQ3 parametrization, " $Q 2$ " $=M^{2}$ ), we get the following result: The largest of the $G \pi^{0}$ production cross sections, namely that for $G=f_{2}(1720)$ with " $L=2$ ", is about one order of magnitude smaller than that obtained for $\pi^{0} \pi^{0}$ production; more precisely, the corresponding ratio is 0.42 with the asymptotic pion distribution amplitude, and 0.063 with the $\mathrm{CZ}$ one. Comparing, on the other hand, the reactions $p \bar{p} \rightarrow G \pi^{ \pm} X$ and $p \bar{p} \rightarrow \pi^{+} \pi^{-} X$, the ratios of the corresponding yields are about half of those obtained in the previous case. Finally, comparing $p \bar{p} \rightarrow G \pi^{0} X$ or $p \bar{p} \rightarrow G \pi^{ \pm} X$ (still for the same choice of $\mathrm{G}$ ) with $p \bar{p} \rightarrow \pi^{ \pm} \pi^{0} X$, the cross sections computed are roughly of the same order (it is to be noticed that in both reactions no gluon-gluon interaction does contribute).

(ix) As compared with $p \bar{p} \rightarrow G \pi^{0} X$, the reactions $p \bar{p} \rightarrow G \eta X$ and $p \bar{p} \rightarrow G \eta^{\prime} X$ would certainly be more promising from a quantitative point of view, since they would involve the contribution of gluon-gluon interactions; that contribution may indeed be expected to increase the $p_{T}$ spectra and the corresponding integrated cross sections by several orders of magnitude (see [2]).

(x) Finally let us remark that, if one of the glueballs here considered contains an admixture of a $q \bar{q}$ state (indeed such admixtures are sometimes advocated for in the theoretical literature, see e.g. [16]), the cross section for $G \pi^{0}$ production might be substantially increased since in this case the gluon-gluon interaction would contribute here as well.

\section{ACKNOWLEDGMENTS}

One of us (F.M.) wishes to thank the Laboratoire de Physique Corpusculaire of the Collège de France, especially its Director Prof. M. Froissart and the Theory Group, for the hospitality extended to him for six weeks in summer 1993; he also acknowledges the support received from the MURST (Ministero dell'Università e della Ricerca Scientifica e Tecnologica, Italy).

This work has been partially supported by the EU program "Human Capital and Mobility" under contract CHRX-CT94-0450. 


\section{APPENDIX A: EXPRESSIONS OF FOUR-MOMENTA, POLARIZATION FOUR-VECTORS AND PROJECTORS OF SPINOR PAIRS}

For the four-momenta of initial partons and final particles involved in the hard process,

defining them in the center-of-mass frame of that process (see Fig. 2a), we use the following expressions (components $0 ; x ; y ; z$ in that order):

$$
\begin{array}{ll}
q^{\mu}=\frac{E}{2}\left(\begin{array}{c}
1 \\
-\sin \Theta \\
0 \\
\cos \Theta
\end{array}\right) & ; \quad \bar{q}^{\mu}=\frac{E}{2}\left(\begin{array}{c}
1 \\
\sin \Theta \\
0 \\
-\cos \Theta
\end{array}\right) \\
G^{\mu}=\frac{E}{2}\left(\begin{array}{c}
1+\eta^{2} \\
0 \\
0 \\
-1+\eta^{2}
\end{array}\right) \quad ; \quad p_{\pi}^{\mu}=\frac{E}{2}\left(1-\eta^{2}\right)\left(\begin{array}{l}
1 \\
0 \\
0 \\
1
\end{array}\right)
\end{array}
$$

while for the four-momenta of the intermediate quarks $q^{\prime}, \bar{q}^{\prime}$ we simply have

$$
q^{\prime}=z p_{\pi} \quad, \quad \bar{q}^{\prime}=(1-z) p_{\pi}
$$

Here we have called all four-momenta like the corresponding particles (except for the pion), and we have defined: $\eta=M / E$.

For the four-momenta of the intermediate gluons $g_{1}, g_{2}$ and for their polarization fourvectors $\epsilon_{1, \lambda_{1}}^{*}, \epsilon_{2, \lambda_{2}}^{*}\left(\lambda_{1,2}=0, \pm 1\right)$, we get, after performing a Lorentz transformation from the glueball rest frame (see Fig. 2 $b$ ) to the c.m. frame of the hard process ( Fig. 2a), the following expressions:

$$
\begin{gathered}
g_{1}^{\mu}=\frac{E}{4}\left(\begin{array}{c}
1+\eta^{2}-\left(1-\eta^{2}\right) \beta \cos \theta \\
2 \eta \beta \sin \theta \cos \varphi \\
2 \eta \beta \sin \theta \sin \varphi \\
-1+\eta^{2}+\left(1+\eta^{2}\right) \beta \cos \theta
\end{array}\right) \quad ; \quad g_{2}=g_{1}(\beta \rightarrow-\beta) \\
\epsilon_{1, \pm 1}^{*}=\epsilon_{2, \mp 1}^{*}=\mp \frac{1}{\sqrt{2}}\left(\epsilon_{1 x} \mp i \epsilon_{1 y}\right)
\end{gathered}
$$

with

$$
\epsilon_{1 x}^{\mu}=\frac{1}{2 \eta}\left(\begin{array}{c}
\left(1-\eta^{2}\right) \sin \theta \\
2 \eta \cos \theta \cos \varphi \\
2 \eta \cos \theta \sin \varphi \\
-\left(1+\eta^{2}\right) \sin \theta
\end{array}\right) \quad ; \quad \epsilon_{1 y}^{\mu}=\left(\begin{array}{c}
0 \\
-\sin \varphi \\
\cos \varphi \\
0
\end{array}\right)
$$

and finally

$$
\epsilon_{1,0}^{\mu}=\frac{1}{\sqrt{1-\beta^{2}}} \frac{1}{2 \eta}\left(\begin{array}{c}
\beta\left(1+\eta^{2}\right)-\left(1-\eta^{2}\right) \cos \theta \\
2 \eta \sin \theta \cos \varphi \\
2 \eta \sin \theta \sin \varphi \\
-\beta\left(1-\eta^{2}\right)+\left(1+\eta^{2}\right) \cos \theta
\end{array}\right) \quad ; \quad \epsilon_{2,0}=\epsilon_{1,0}(\beta \rightarrow-\beta)
$$


As for the projectors of the spinor pair corresponding to $q^{\prime}, \bar{q}^{\prime}$, we make the substitution (in accordance with Ref. [11], accounting for the fact that the $\left(q^{\prime}, \bar{q}^{\prime}\right)$ system is in a pseudoscalar state):

$$
\left(v_{\bar{q}^{\prime}} \bar{u}_{q^{\prime}}\right)_{P S}=\frac{1}{\sqrt{2}}\left(v_{\bar{q}^{\prime}}^{\uparrow} \bar{u}_{q^{\prime}}^{\downarrow}-v_{\bar{q}^{\prime}}^{\downarrow} \bar{u}_{q^{\prime}}^{\uparrow}\right) \rightarrow \frac{1}{\sqrt{2}} \gamma_{5} \not \phi_{\pi}
$$

On the other hand, for the spinor pair corresponding to the incoming quarks we use:

$$
u_{q}^{\uparrow} \bar{v}_{\bar{q}}^{\downarrow}=-\frac{E}{2 \sqrt{2}} \phi_{+}\left(1-\gamma_{5}\right)
$$

with

$$
\epsilon_{+}^{\mu}=-\frac{1}{\sqrt{2}}\left(\begin{array}{c}
0 \\
\cos \Theta \\
i \\
\sin \Theta
\end{array}\right)
$$

noticing that no other helicity states of the incoming quarks need to be considered in the calculation (see section III).

As specified in section II, we let $\eta$ go to zero; this is done, precisely, once we have computed the helicity amplitudes of the subprocess $q \bar{q} \rightarrow g_{1} g_{2}\left(q^{\prime} \bar{q}^{\prime}\right)_{P S}$. Then all divergences in $\eta$, due to the $\eta^{-1}$ factors appearing in the expressions of the polarization four-vectors of the gluons (see Eqs. (A6), (A7)) must vanish. This (as well as the vanishing of all divergences in $\beta$ after applying Eq. (11)) is a good check of the correctness of the calculation.

\section{APPENDIX B: HELICITY AMPLITUDES FOR THE PROCESSES $q \bar{q} \rightarrow G\left(q^{\prime} \bar{q}^{\prime}\right)_{P S}$ AND $q \bar{q} \rightarrow G \pi$}

In this appendix we present the expressions of all independent helicity amplitudes for the process $q \bar{q} \rightarrow G\left(q^{\prime} \bar{q}^{\prime}\right)_{P S}$, i.e. $\mathcal{M}_{q \bar{q} \rightarrow G\left(q^{\prime} \bar{q}^{\prime}\right)_{P S}}^{\lambda \bar{\lambda}, \Lambda}(E, \Theta, z)$, before their convolution with the pion distribution amplitude (see Eq. (任)), but with the final quark-antiquark pair being specified to be in a pseudoscalar spin state. In each case, the corresponding helicity amplitudes for the process $q \bar{q} \rightarrow G \pi$, i.e. $\mathcal{M}_{q \bar{q} \rightarrow G \pi}^{\lambda \bar{\lambda}, \Lambda}(E, \Theta)$, after convolution with the asymptotic resp. Chernyak-Zhitnitsky pion distribution amplitude are also given. All helicity amplitudes not explicitly shown here can be derived by means of symmetry properties (see section II) from those given hereafter, or are vanishing. We have fixed the helicities of the initial quark-antiquark pair as follows: $\lambda=1 / 2, \bar{\lambda}=-1 / 2$. For shortness, we use the following notation: $\mathcal{M}_{P S}^{\Lambda}$ for $\mathcal{M}_{\left.q \bar{q} \rightarrow G\left(q^{\prime}\right)^{\prime}\right)_{P S}}^{\lambda \bar{\lambda}, \Lambda}(E, \Theta, z)$, and $\mathcal{M}_{(a s)}^{\Lambda}$ resp. $\mathcal{M}_{(C Z)}^{\Lambda}$ for the amplitudes obtained after convolution of $\mathcal{M}_{P S}^{\Lambda}$ with the asymptotic resp. the Chernyak-Zhitnitsky pion distribution amplitude. Furthermore we use $c=\cos \Theta, s=\sin \Theta, u=z(1-z), w=2 z-1$, $\mathcal{L}_{c}=\ln [(1+\cos \Theta) /(1-\cos \Theta)]$. The constant $f_{L}$ has been defined in Eq. (3). Finally, as usual, $g_{s}=\sqrt{4 \pi \alpha_{s}}$, while $i, j$ are the color indices of the incoming quark and antiquark respectively. 
(i) $L=S=J=0$

$$
\begin{aligned}
\mathcal{M}_{P S}^{0} & =-\frac{8}{27} g_{s}^{4} f_{0} \delta_{i j} \frac{1}{E^{2} s^{3}} \frac{-8 s^{4}+s^{2}(-96 u+25)+64 u}{u(1-c w)} \\
\mathcal{M}_{(a s)}^{0} & =-\frac{8}{9 \sqrt{3}} g_{s}^{4} f_{0} f_{\pi} \delta_{i j} \frac{1}{E^{2} s^{3}}\left\{24-\frac{8}{c^{2}}+\frac{1-c^{2}}{2 c^{3}}\left(8-7 c^{2}+8 c^{4}\right) \mathcal{L}_{c}\right\} \\
\mathcal{M}_{(C Z)}^{0} & =-\frac{40}{9 \sqrt{3}} g_{s}^{4} f_{0} f_{\pi} \delta_{i j} \frac{1}{E^{2} s^{3}}\left\{8 c^{2}-7+\frac{37}{3 c^{2}}-\frac{8}{c^{4}}+\frac{1-c^{2}}{2 c^{5}}\left(8-7 c^{2}+8 c^{4}\right) \mathcal{L}_{c}\right\}
\end{aligned}
$$

(ii) $L=S=1, J=0$

$$
\begin{gathered}
\mathcal{M}_{P S}^{0}=-\frac{8 \sqrt{2}}{81} g_{s}^{4} f_{1} \delta_{i j} \frac{1}{E^{2} s^{3}} \frac{1}{u(1-c w)^{2}} \\
\times\left\{s^{4}(4 c+5 w)+4 s^{2}[c(21 u-4)+4 w(5 u+1)]+64 u(c-w)\right\} \\
\mathcal{M}_{(a s)}^{0}=-\frac{8 \sqrt{2}}{27 \sqrt{3}} g_{s}^{4} f_{1} f_{\pi} \delta_{i j} \frac{1}{E^{2} s^{3}}\left\{-4 c^{3}+25 c+\frac{7}{c}-\frac{12}{c^{3}}\right. \\
\left.+\frac{1-c^{2}}{2 c^{4}}\left(12+c^{2}+5 c^{4}\right) \mathcal{L}_{c}\right\} \\
\mathcal{M}_{(C Z)}^{0}=-\frac{40 \sqrt{2}}{27 \sqrt{3}} g_{s}^{4} f_{1} f_{\pi} \delta_{i j} \frac{1}{E^{2} s^{3}}\left\{4 c^{3}+\frac{1}{c}+\frac{61}{3 c^{3}}-\frac{20}{c^{5}}\right. \\
\left.+\frac{1-c^{2}}{2 c^{6}}\left(20-7 c^{2}-3 c^{4}+8 c^{6}\right) \mathcal{L}_{c}\right\}
\end{gathered}
$$

(iii) $L=0, S=2, J=2$

$$
\begin{aligned}
\mathcal{M}_{P S}^{ \pm 2} & =\frac{512}{9 \sqrt{3}} g_{s}^{4} f_{0} \delta_{i j} \frac{1}{E^{2} s(1 \pm c)} \frac{1}{(1 \mp w)} \\
\mathcal{M}_{(a s)}^{ \pm 2} & =\frac{128}{9} g_{s}^{4} f_{0} f_{\pi} \delta_{i j} \frac{1}{E^{2} s(1 \pm c)} \\
\mathcal{M}_{(C Z)}^{ \pm 2} & =\frac{5}{3} \mathcal{M}_{(a s)}^{ \pm 2}
\end{aligned}
$$

$$
\begin{gathered}
\mathcal{M}_{P S}^{0}=-\frac{8 \sqrt{2}}{27} g_{s}^{4} f_{0} \delta_{i j} \frac{1}{E^{2} s^{3}} \frac{-4 s^{4}-s^{2}(48 u+1)+32 u}{u(1-c w)} \\
\mathcal{M}_{(a s)}^{0}=-\frac{8 \sqrt{2}}{9 \sqrt{3}} g_{s}^{4} f_{0} f_{\pi} \delta_{i j} \frac{1}{E^{2} s^{3}}\left\{12-\frac{4}{c^{2}}+\frac{1-c^{2}}{2 c^{3}}\left(4-c^{2}\right)\left(1-4 c^{2}\right) \mathcal{L}_{c}\right\} \\
\mathcal{M}_{(C Z)}^{0}=-\frac{40 \sqrt{2}}{9 \sqrt{3}} g_{s}^{4} f_{0} f_{\pi} \delta_{i j} \frac{1}{E^{2} s^{3}}\left\{4 c^{2}-17+\frac{59}{3 c^{2}}-\frac{4}{c^{4}}\right. \\
\left.+\frac{1-c^{2}}{2 c^{5}}\left(4-c^{2}\right)\left(1-4 c^{2}\right) \mathcal{L}_{c}\right\}
\end{gathered}
$$


(iv) $L=2, S=0, J=2$

$$
\begin{aligned}
& \mathcal{M}_{P S}^{ \pm 2}= \frac{1024 \sqrt{2}}{135 \sqrt{3}} g_{s}^{4} f_{2} \delta_{i j} \frac{1}{E^{2} s(1 \pm c)} \frac{1}{1 \mp w} \\
& \mathcal{M}_{(a s)}^{ \pm 2}= \frac{256 \sqrt{2}}{135} g_{s}^{4} f_{2} f_{\pi} \delta_{i j} \frac{1}{E^{2} s(1 \pm c)} \\
& \mathcal{M}_{(C Z)}^{ \pm 2}= \frac{5}{3} \mathcal{M}_{(a s)}^{ \pm 2} \\
& \mathcal{M}_{P S}^{0}=-\frac{32}{405} g_{s}^{4} f_{2} \delta_{i j} \frac{1}{E^{2} s^{3}} \frac{1}{u(1-c w)^{3}} \\
& \times\left\{-8 s^{6}(2 u-1)+s^{4}[7 c w+2 u(-224 u+137)-40]\right. \\
&\left.+s^{2}[22 c w(10 u-3)+448 u(2 u-1)+66]-192 u(c w+2 u-1)\right\} \\
& \mathcal{M}_{(a s)}^{0}=-\frac{32}{135 \sqrt{3}} g_{s}^{4} f_{2} f_{\pi} \delta_{i j} \frac{1}{E^{2} s^{3}}\left\{-5 c^{2}+\frac{63}{2}+\frac{43}{2 c^{2}}\right. \\
&\left.\quad+\frac{1-c^{2}}{4 c^{5}}\left(48-11 c^{2}+9 c^{4}+8 c^{6}\right) \mathcal{L}_{c}\right\} \\
& \mathcal{M}_{(C Z)}^{0}=-\frac{32}{27 \sqrt{3}} g_{s}^{4} f_{2} f_{\pi} \delta_{i j} \frac{1}{E^{2} s^{3}}\left\{-3 c^{2}+\frac{193}{6}-\frac{373}{6 c^{2}}+\frac{101}{c^{4}}-\frac{60}{c^{6}}\right. \\
&\left.\quad+\frac{1-c^{2}}{4 c^{7}}\left(120-122 c^{2}+59 c^{4}-11 c^{6}+8 c^{8}\right) \mathcal{L}_{c}\right\}
\end{aligned}
$$

(v) $L=S=J=2$

$$
\begin{aligned}
\mathcal{M}_{P S}^{ \pm 2}= & \frac{256 \sqrt{2}}{135 \sqrt{21}} g_{s}^{4} f_{2} \delta_{i j} \frac{1}{E^{2} s(1 \pm c)} \frac{1}{1 \mp w} \\
\mathcal{M}_{(a s)}^{ \pm 2}= & \frac{64 \sqrt{2}}{135 \sqrt{7}} g_{s}^{4} f_{2} f_{\pi} \delta_{i j} \frac{1}{E^{2} s(1 \pm c)} \\
\mathcal{M}_{(C Z)}^{ \pm 2}= & \frac{5}{3} \mathcal{M}_{(a s)}^{ \pm 2} \\
\mathcal{M}_{P S}^{0}= & \frac{8}{405 \sqrt{7}} g_{s}^{4} f_{2} \delta_{i j} \frac{1}{E^{2} s^{3}} \frac{1}{u(1-c w)^{3}}\left\{4 s^{6}(68 u-13)\right. \\
& +s^{4}[-140 c w+4 u(560 u-311)+311]+2 s^{2}[-c w(568 u-171) \\
& +2 u(-784 u+527)-171]+576 u(c w+2 u-1)\} \\
\mathcal{M}_{(a s)}^{0}= & \frac{8 \sqrt{3}}{405 \sqrt{7}} g_{s}^{4} f_{2} f_{\pi} \delta_{i j} \frac{1}{E^{2} s^{3}}\left\{-20 c^{2}-180+\frac{224}{c^{2}}-\frac{96}{c^{4}}\right.
\end{aligned}
$$




$$
\begin{array}{r}
\mathcal{M}_{(C Z)}^{0}=\frac{8 \sqrt{3}}{81 \sqrt{7}} g_{s}^{4} f_{2} f_{\pi} \delta_{i j} \frac{1}{E^{2} s^{3}}\left\{-96 c^{2}+\frac{1295}{3}-\frac{2795}{3 c^{2}}+\frac{812}{c^{4}}-\frac{240}{c^{6}}\right. \\
\left.+\frac{1-c^{2}}{2 c^{7}}\left(240-652 c^{2}+529 c^{4}-160 c^{6}+16 c^{8}\right) \mathcal{L}_{c}\right\}
\end{array}
$$

(vi) $L=S=2, J=4$

$$
\begin{aligned}
\mathcal{M}_{P S}^{ \pm 2}= & \frac{1024 \sqrt{2}}{135 \sqrt{7}} g_{s}^{4} f_{2} \delta_{i j} \frac{1}{E^{2} s(1 \pm c)} \frac{1}{1 \mp w} \\
\mathcal{M}_{(a s)}^{ \pm 2}= & \frac{256 \sqrt{2}}{45 \sqrt{21}} g_{s}^{4} f_{2} f_{\pi} \delta_{i j} \frac{1}{E^{2} s(1 \pm c)} \\
\mathcal{M}_{(C Z)=}^{ \pm 2}= & \frac{5}{3} \mathcal{M}_{(a s)}^{ \pm 2} \\
\mathcal{M}_{P S}^{0}= & \frac{32}{135 \sqrt{35}} g_{s}^{4} f_{2} \delta_{i j} \frac{1}{E^{2} s^{3}} \frac{1}{u(1-c w)^{3}} \\
\times & \left\{8 s^{6}(2 u-1)+s^{4}[-7 c w+64 u(7 u-4)+22]\right. \\
& \left.+s^{2}[10 c w(-22 u+3)+8 u(-112 u+47)-30]+192 u(c w+2 u-1)\right\} \\
\mathcal{M}_{(a s)}^{0}= & \frac{32 \sqrt{3}}{135 \sqrt{35}} g_{s}^{4} f_{2} f_{\pi} \delta_{i j} \frac{1}{E^{2} s^{3}}\left\{5 c^{2}-18-\frac{35}{c^{2}}+\frac{24}{c^{4}}\right. \\
\mathcal{M}_{(C Z)}^{0}= & \left.\quad-\frac{1-c^{2}}{2 c^{5}}\left(24-19 c^{2}+4 c^{6}\right) \mathcal{L}_{c}\right\} \\
27 \sqrt{35} & g_{s}^{4} f_{2} f_{\pi} \delta_{i j} f_{\pi} \frac{1}{E^{2} s^{3}}\left\{3 c^{2}-\frac{245}{3}+\frac{578}{3 c^{2}}-\frac{182}{c^{4}}+\frac{60}{c^{6}}\right. \\
& \left.\quad-\frac{1-c^{2}}{2 c^{7}}\left(60-142 c^{2}+106 c^{4}-19 c^{6}+4 c^{8}\right) \mathcal{L}_{c}\right\}
\end{aligned}
$$




\section{REFERENCES}

[1] E.H. Kada, P. Kessler, and J. Parisi, Phys. Rev D 39, 2657 (1989).

[2] L. Houra-Yaou, P. Kessler, and J. Parisi, Phys. Rev. D 45, 794 (1992).

[3] A. Ichola and J. Parisi, Z. Phys. C 66, 653 (1995).

[4] H. Fritzsch and M. Gell-Mann, in "Proceedings of the XVI International Conference on High-Energy Physics", Chicago, Illinois, 1973, eds. J.S. Jackson and A. Roberts (NAL, Batavia, 1973), Vol. 2, p. 135.

[5] See e.g. A. Palano, Report CERN-EP/87-92, 1987.

[6] C.A. Heusch, "Status of gluonium searches", Report CERN PPE/91-225.

[7] G. Eigen, in "Proceedings of the IX International Workshop on Photon-Photon Collisions", San Diego, California, 1992, eds. D.O. Caldwell and H.P. Paar (World Scientific, Singapore, 1992), p. 283.

[8] See also Particle Data Group, L. Montanet et al., Phys. Rev. D 50 3-I (1994).

[9] A. Palano, in same Proceedings as in Ref. [7], p. 308.

[10] K.T. Chao, " $\xi(2230)$ is likely to be a glueball", Report PUTP 94-26.

[11] G.P. Lepage and S.J. Brodsky, Phys. Rev. D 22, 2157 (1980); S.J. Brodsky and G.P. Lepage, ibid., 24, 1808 (1981); 24, 2948 (1981).

[12] V.L. Chernyak and A.R. Zhitnitsky, Nucl. Phys. B 201, 492 (1982).

[13] CTEQ Collaboration, H. Lai et al., "Global QCD analysis and the CTEQ parton distributions", Report MSU-HEP-41024, CTEQ-404 (1994).

[14] A.D. Martin, W.J. Stirling, and R.G. Roberts, Phys. Rev. D 50, 6734 (1994).

[15] K. Djagouri, J.J. Dugne, C. Carimalo, and P. Kessler, Z. Phys. C 45, 267 (1989).

[16] C. Amsler and F. Close, Phys. Lett. B 353, 385 (1995). 


\section{FIGURES}

FIG. 1. The Feynman graphs that, to lowest order in QCD, contribute to the process $q \bar{q} \rightarrow g_{1} g_{2} q^{\prime} \bar{q}^{\prime}$. The graphs are grouped according to their color factors (a convolution with the color part of the glueball and the pion wave function is understood): a) $c_{F}=8 \delta_{i j} /(9 \sqrt{6})$; b) $c_{F}=-\delta_{i j} /(9 \sqrt{6})$; c1) $c_{F}=i \delta_{i j} / \sqrt{6}$; c2) $c_{F}=-i \delta_{i j} / \sqrt{6}$; c3) $c_{F}=-2 \delta_{i j} / \sqrt{6}$. $i, j$ are the color indices of the incoming quark and antiquark, respectively. For each graph, except for that including the four-gluon vertex, the corresponding one where $g_{1}$ and $g_{2}$ are exchanged must be also taken into account.

FIG. 2. Kinematics schemes for: a) the process $q \bar{q} \rightarrow G \pi$ in the center-of-mass frame of $q$ and $\bar{q}$; b) the process $q \bar{q} \rightarrow g_{1} g_{2} \pi$ in the center-of-mass frame of $g_{1}$ and $g_{2}$.

FIG. 3. The transverse-momentum spectrum, multiplied by $p_{T}{ }^{7}$ and $B$, predicted for the reaction $p \bar{p} \rightarrow G \pi^{0} X$ at $s=4 \times 10^{5} \mathrm{GeV}^{2}$. Here $G=\eta(1440)$ and $B=B r\left(\eta(1440) \rightarrow \rho^{0} \rho^{0}\right)$. Both the asymptotic (dashed curve) and the Chernyak-Zhitnitsky (full curve) pion distribution amplitudes have been considered. The parametrization CTEQ3 (at leading order in QCD) [13] for the parton distribution functions has been used.

FIG. 4. Same as Fig. 3, with $G=f_{2}(1720), B=B r\left(f_{2}(1720) \rightarrow K \bar{K}\right)$. The same quantum states as in Refs. [1] 3 are considered for the $f_{2}(1720)$.

FIG. 5. Same as Fig. 3, with $G=X(2220), B=B r\left(X(2220) \rightarrow \pi^{+} \pi^{-}\right)$. The same quantum states as in Refs. [1] 3 are considered for the $X(2220)$.

FIG. 6. Comparison between the results of Fig. 3 and the corresponding ones for the reaction $p \bar{p} \rightarrow G \pi^{ \pm} X$. Same notation as in Fig. 3. In addition, the dot-dashed and dotted curves refer to $\pi^{ \pm}$production, using respectively the $\mathrm{CZ}$ and asymptotic distribution amplitude.

FIG. 7. Comparison between the results of Fig. 3 and the analogous ones, obtained using a different set (MRSA) of parton distribution functions (see Ref. [14]). Same notation as in Fig. 3 . In addition, the dot-dashed and dotted curves correspond to MRSA, using respectively the CZ and asymptotic distribution amplitude.

FIG. 8. Comparison between the results of Fig. 3 and the analogous ones, obtained using a different prescription for the value of " $Q$ " in the expression of both the function $F\left(x, x^{\prime}\right.$, " $Q$ ") (see Eq. (8)) and $\alpha_{s}$. Same notation as in Fig. 3. In addition, the dot-dashed and dotted curves correspond to " $Q$ " $=p_{T}^{2}$ instead of " $Q^{2} "=M^{2}$, using respectively the $\mathrm{CZ}$ and asymptotic distribution amplitude. 

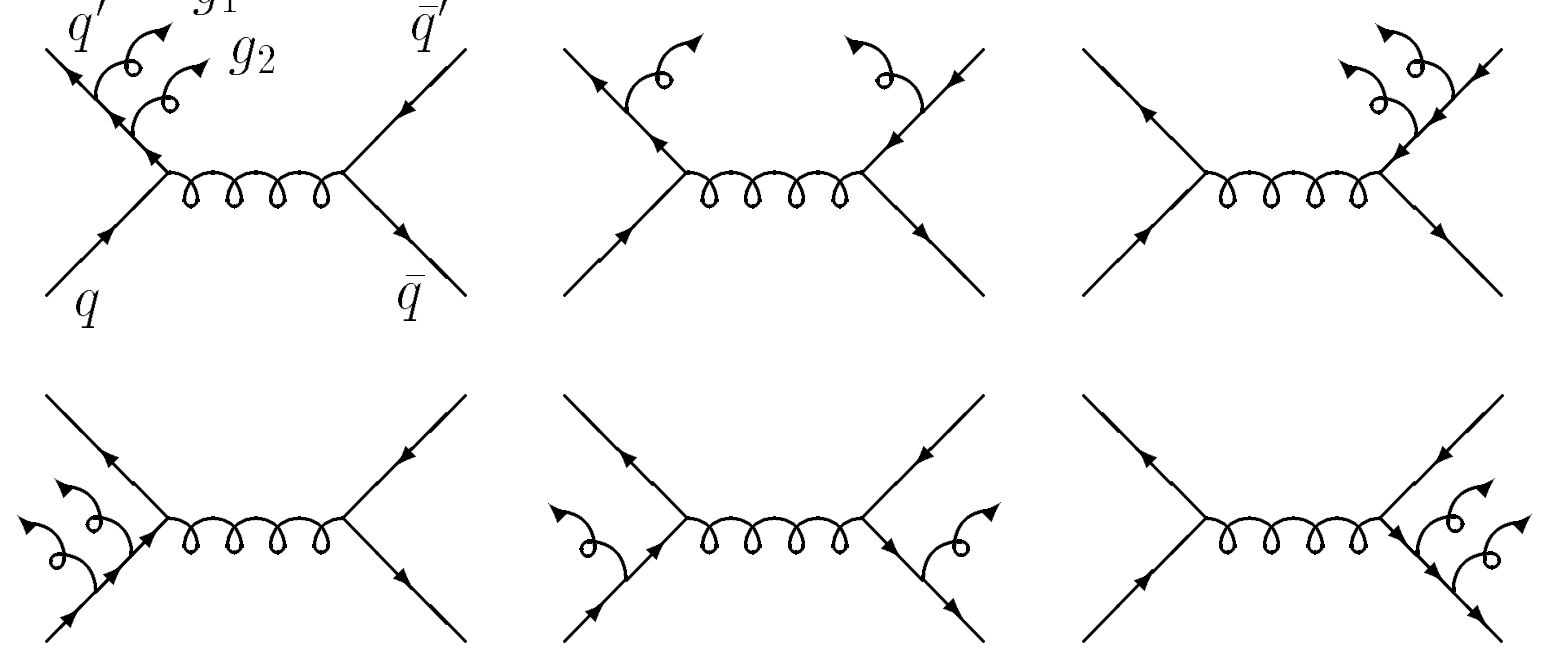

(a)
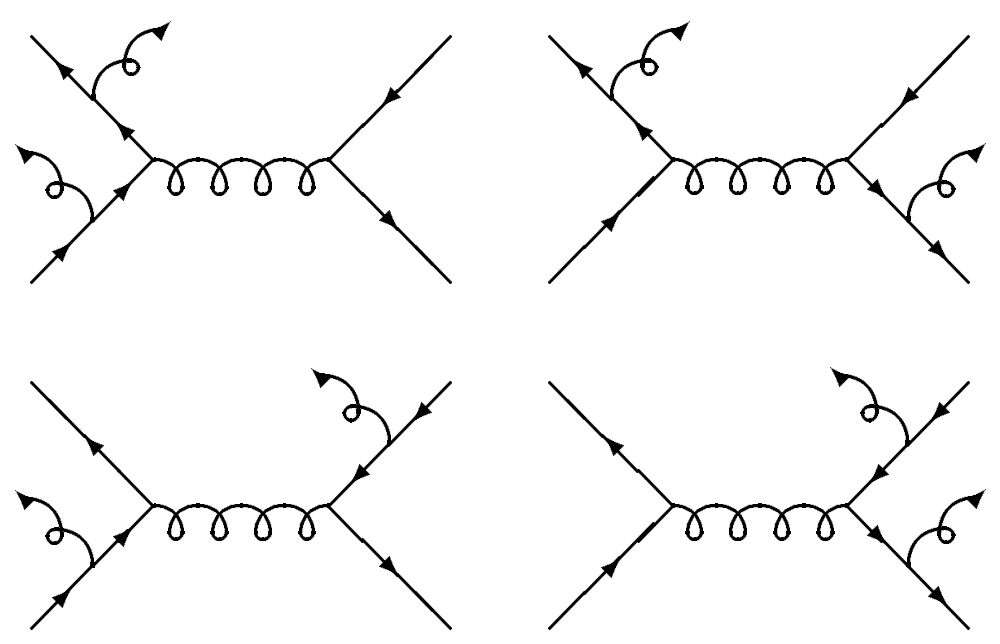

(b)
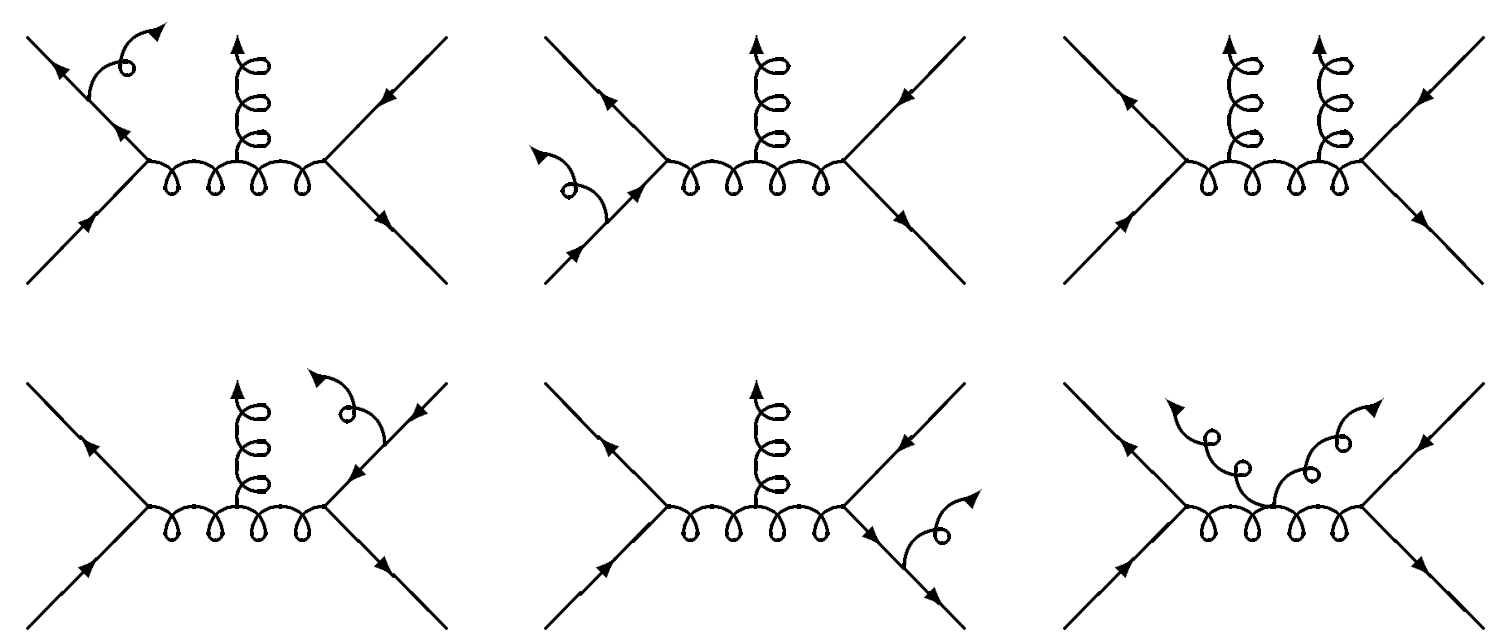

(c1)

(c2)

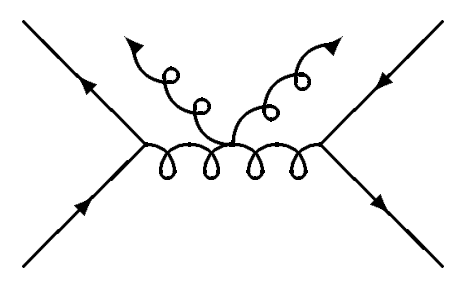

(c3) 

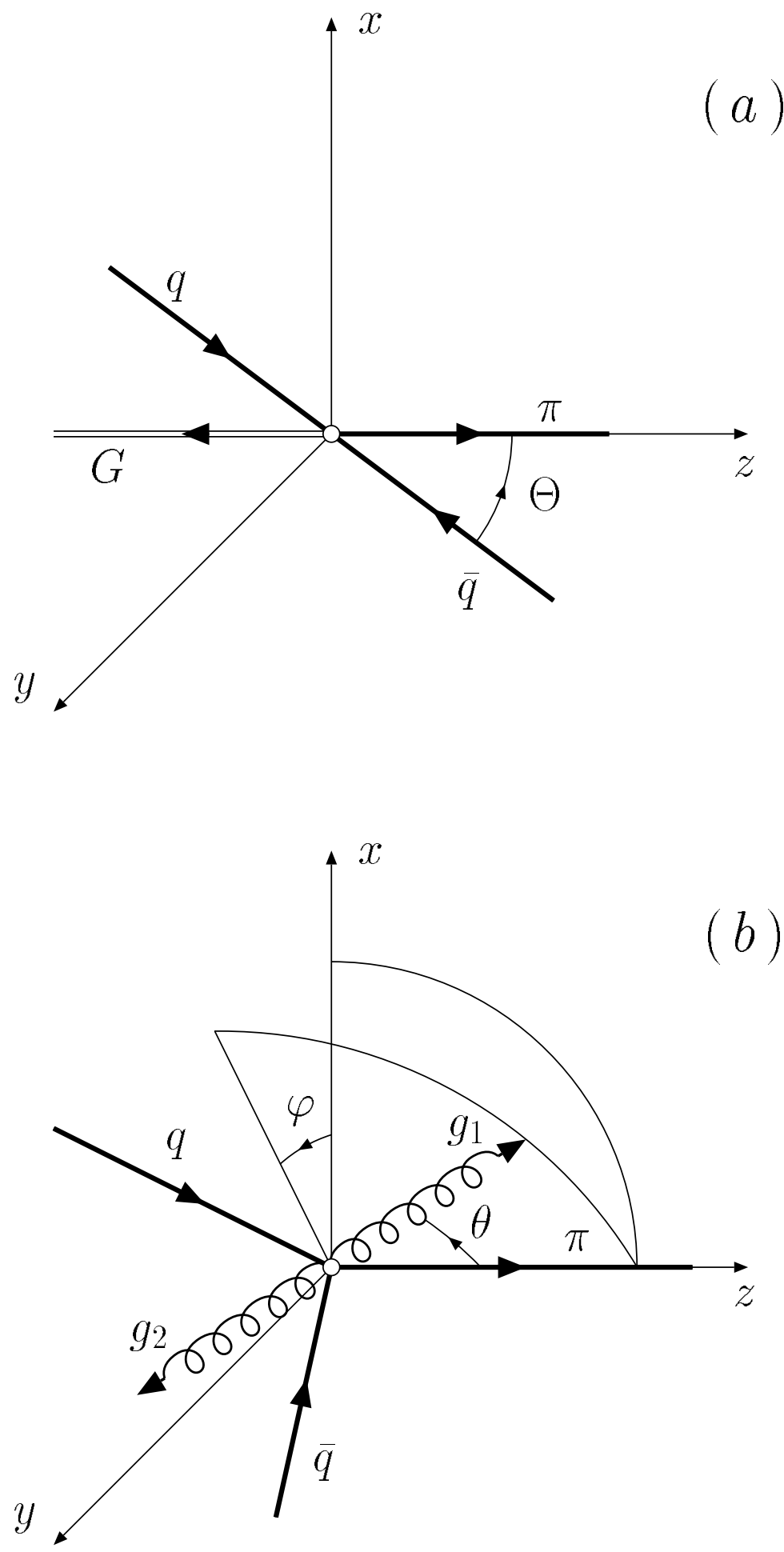

FIG. 2 


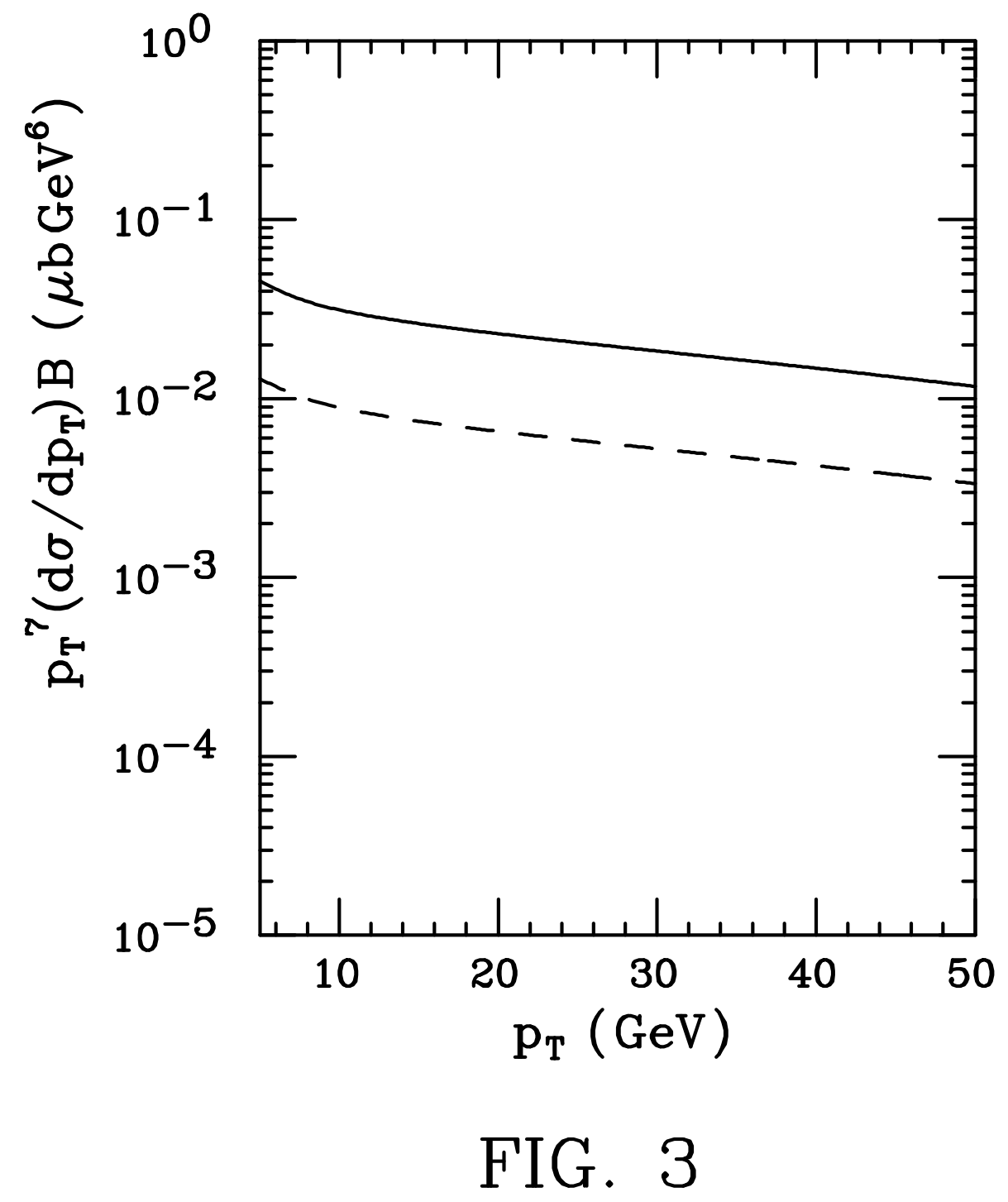




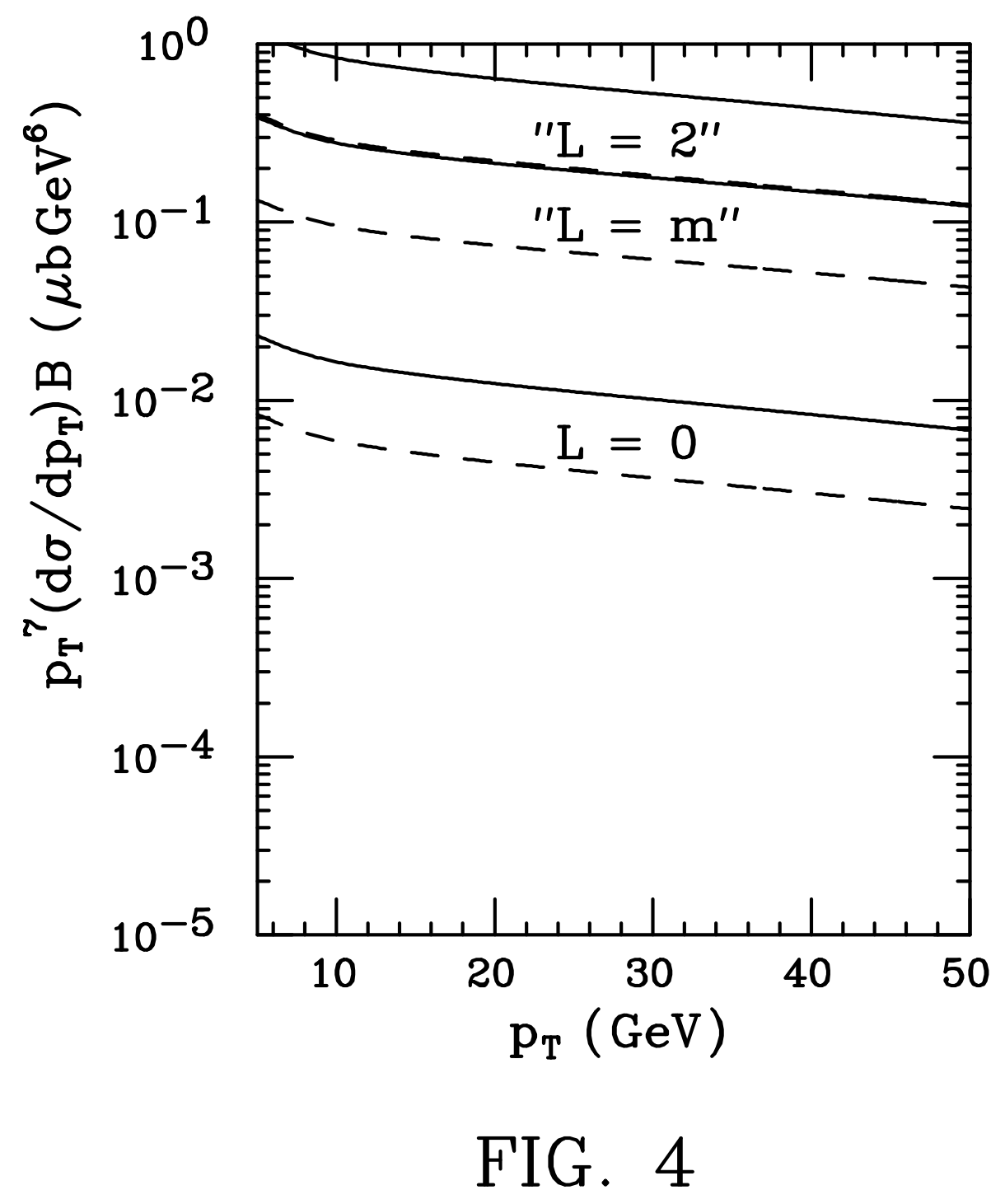




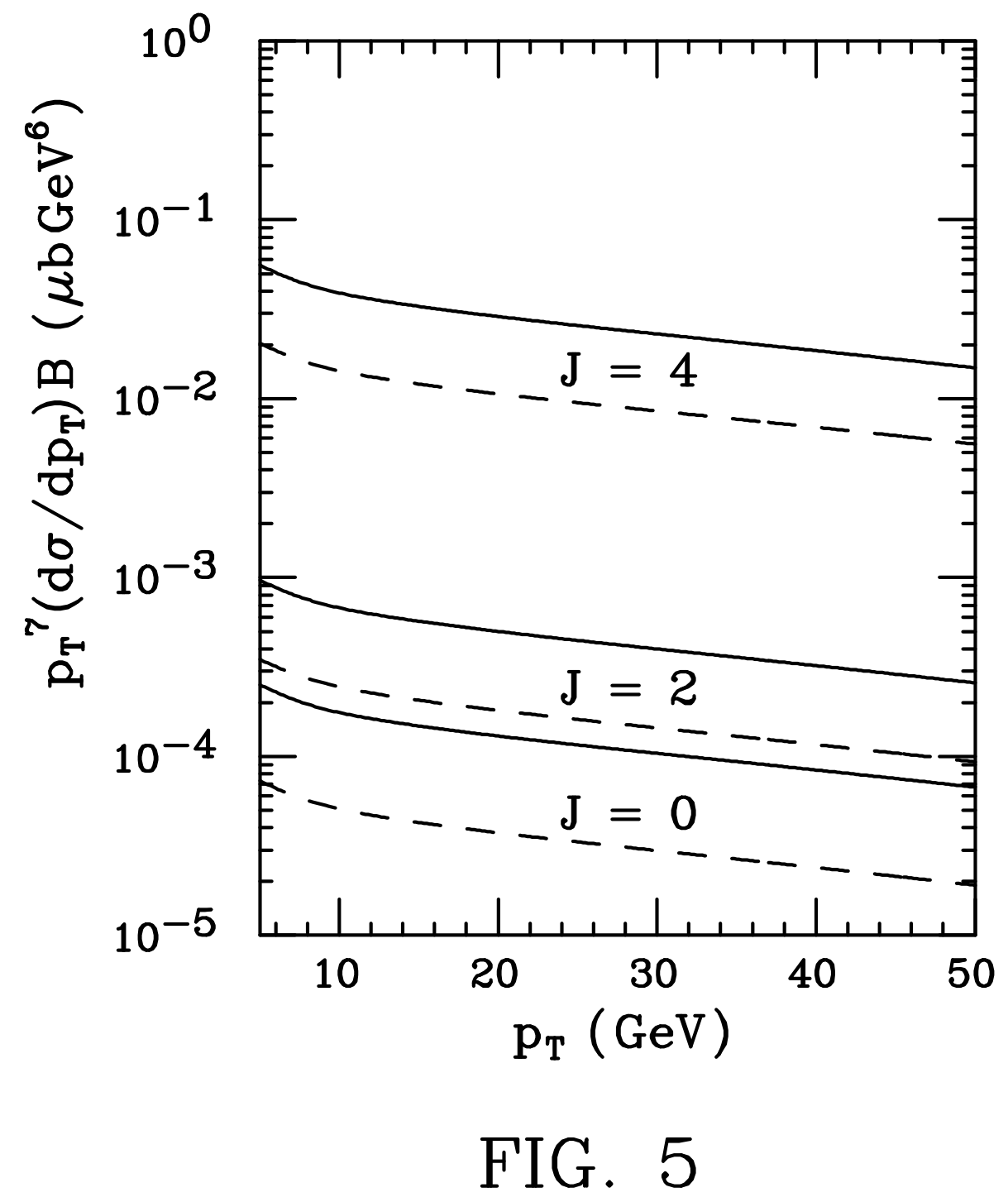




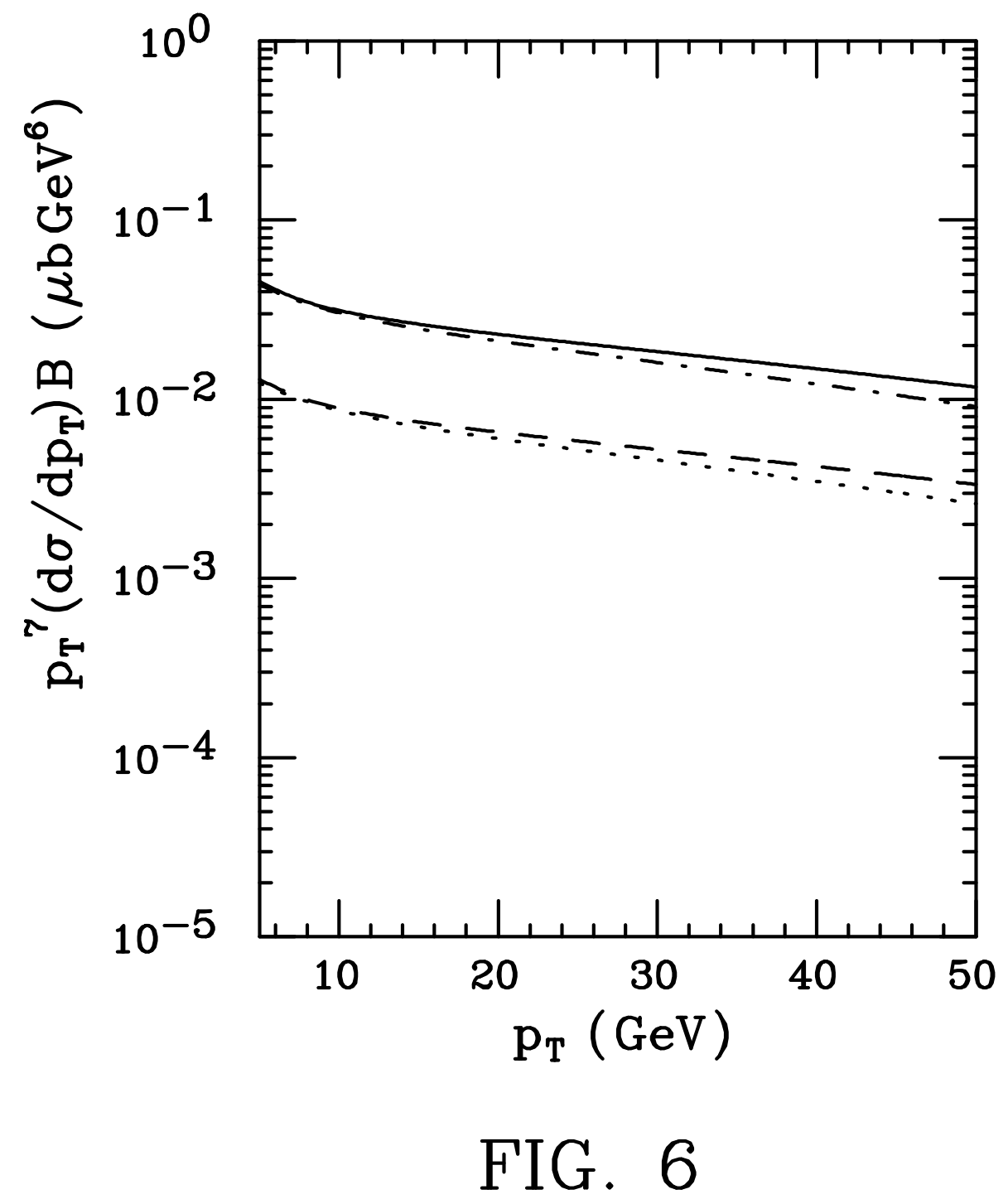




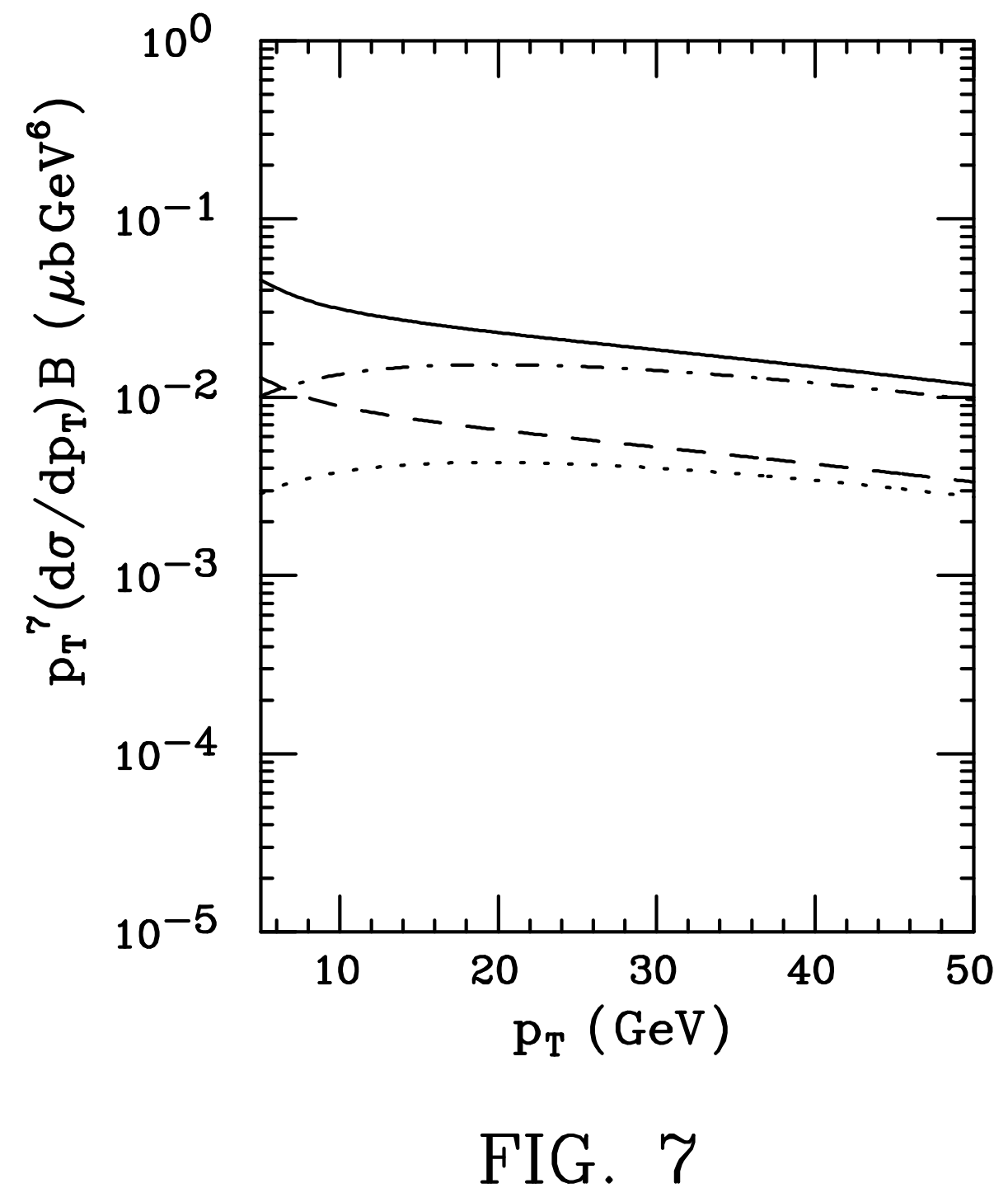




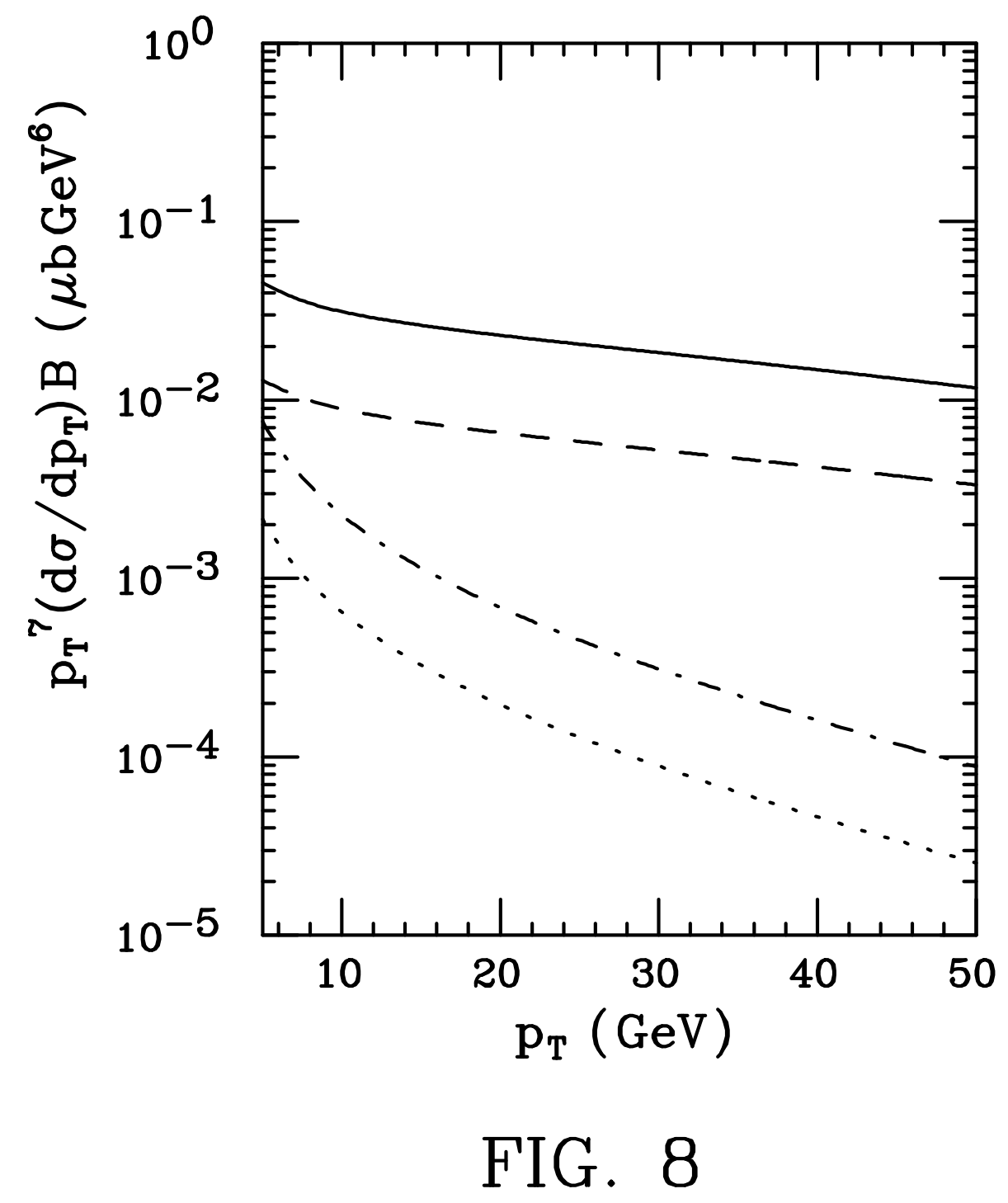

